\title{
Measurement of physical parameters of forest fires by infrared imaging methods
}

\author{
J. M. Aranda, J. Meléndez, A. J. de Castro \& F. López \\ LIR - UC3M: Laboratorio de Sensores Teledetección e Imagen IR \\ Departamento de Física, Universidad Carlos III de Madrid, Spain
}

\begin{abstract}
In the last years, infrared (IR) cameras have been used increasingly in forest fire related applications, but, in contrast with industrial applications of IR thermography, quantitative studies are almost nonexistent. This is due to the complex spectral and spatial structure of the IR emission that prevents a straightforward interpretation of images. However, multispectral systems, even with a resolution of only a few bands, can be used to great advantage in order to classify the infrared scene into different regions (flame, embers, ashes, background), each with a different spectral signature and affected in a different way by the atmosphere. In this work it is shown how this procedure makes possible to take into account spectral structure, in order to measure physical parameters like rate of spread, embers temperature, and radiated power in the spectral range of the camera. Measurements have been obtained in several experimental conditions, and results have been compared to data from estimations derived from standard methods. This comparison demonstrates the consistency of the two approaches, and makes possible to estimate also total radiated power, fire intensity and heat release per unit area from infrared measurements only.
\end{abstract}

Keywords: infrared imaging, multispectral, classification, forest fires.

\section{Introduction}

In the last years, infrared (IR) sensors, operating from satellite, ground-based or airborne platforms, have been used increasingly in forest fire related applications [1], including fire danger estimation, fire detection, fire monitoring, mapping of burned areas and follow-up of after-fire recovery. 
However, most IR applications have limited themselves to fire imaging, not trying to measure the basic fire parameters (rate of spread, flame height, temperature, fire front intensity, etc), which are estimated using video recording, thermocouples and mass loss (burned fuel) measurements. These standard techniques are straightforward for laboratory fires but face difficulties when applied in field conditions: rate of spread and flame height measurements by visual inspection may be impossible because of smoke obscuration (Fig. 1, left); temperature measurements need the previous deployment of a grid of thermocouples; fire front intensity estimation requires to determine the amount of burned fuel by means of pre-burn and post-burn inventories, etc. These procedures are tedious and suitable only for prescribed burns (not for wild fires) and provide only overall data, difficult to correlate with instantaneous parameters like wind velocity and direction.
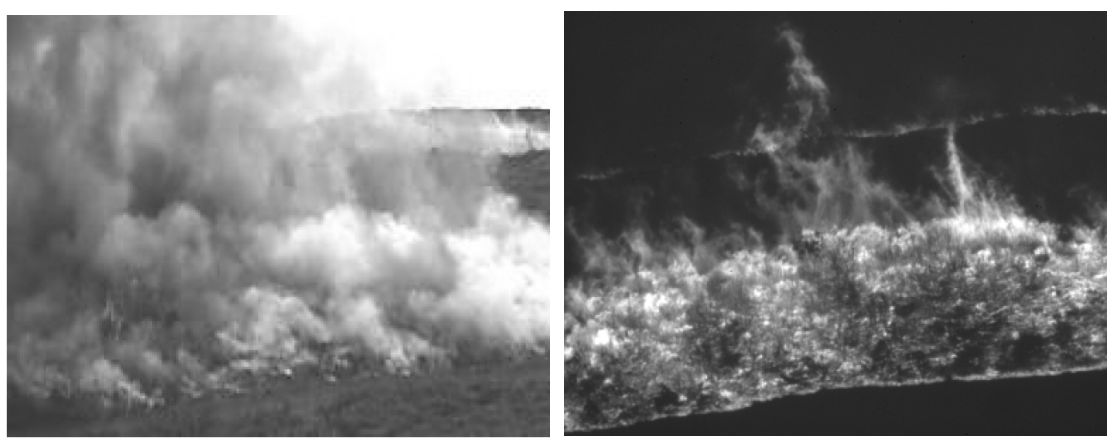

Figure 1: (Left) Visible aspect of a field burn at $220 \mathrm{~m}$. (Right). The same scene in the $3,6 \mu \mathrm{m}$ band. The fire front, propagating upward, is clearly seen.

These drawbacks make IR imaging an appealing alternative [2]. Cameras are well suited for field operation, can visualize easily the fire front through smoke (Fig. 1, right), and, with a proper calibration, they give a measurement of the radiance emitted by each point that can be related to temperature and radiated power, with very good spatial and temporal resolution.

However, there are some difficulties when trying to obtain quantitative measurements with IR imaging techniques that have prevented them from being extensively used. These difficulties stem from the physical properties of forest fires: complex targets with several different regions (flames, fire front, embers, ashes...) changing with time. As explained in section 2, the spectral emission profiles of the regions may be very different from that of a blackbody. This, in turn, means that each region is affected by atmospheric absorption in a different way, and that apparent temperatures may be very different from real temperatures. Thus, in addition to radiometric calibration, a scene model is necessary to obtain quantitative measurements.

In this work such a scene analysis is made by using multi-spectral images. In section 3 it is shown that classification techniques, a standard tool for the 
analysis of satellite multi-spectral images, provide a method to distinguish different fire regions. This makes possible a region-specific processing, which is the key to overcome many of the difficulties posed by quantitative measurements in forest fires, as explained in section 4 (for rate of spread and temperature measurements) and section 5 (for radiated power measurements).

\section{Spectral properties of fire emission and atmospheric transmission}

In a fire the solid components (embers, ashes) and gaseous components (flame) have very different spectral emission profiles. The contrast can be appreciated in Fig. 2, which shows two spectra acquired with a Fourier Transform spectroradiometer in an experimental forest fire at a distance of $3 \mathrm{~m}$, pointing to flames (left) and to nonflaming embers (right). The vertical axis represents spectral radiance in arbitrary units. Flame emission is dominated by a strong peak due to $\mathrm{CO}_{2}$ emission, with a weak continuum emission in the whole spectral region. In contrast, the emission of hot embers shows a blackbody profile, altered only by the absorption due to atmospheric $\mathrm{CO}_{2}$ and $\mathrm{H}_{2} \mathrm{O}$.

A calibrated IR camera provides a measurement of the incoming radiance, integrated over its spectral interval of operation. In order to minimize the effects of atmospheric absorption, most IR cameras operate in a high transmittance "atmospheric window", either the medium IR band (MIR, from 3 to $5 \mu \mathrm{m})$ or the thermal IR band (TIR, from 8 to $12 \mu \mathrm{m})$.
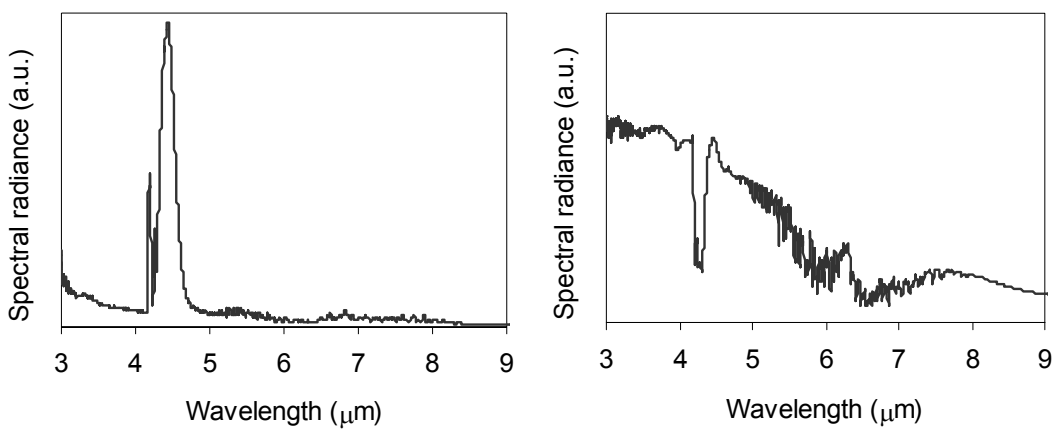

Figure 2: Spectral radiance emitted by two different regions of a experimental forest fire: (left) flames, (right) embers without flame. Spectra were measured with a Fourier Transform Infrared spectroradiometer.

The strong spectral structure of atmospheric transmittance means that its effective value will be very different for different spectral bands, but also that even in the same spectral band effective transmittances will differ for different emitters: in order to take into account properly the atmospheric effects on the signal of a particular pixel, it must be known to which fire region it belongs. 
In other words, it will be necessary to classify the scene. For the $r$ band, the relationship between radiance emitted by the fire and radiance incoming to the camera will be:

$$
\mathrm{L}_{\mathrm{in}}^{\mathrm{i}, \mathrm{r}}=\tau^{\mathrm{i}, \mathrm{r}} \cdot \mathrm{L}_{\mathrm{em}}^{\mathrm{i}, \mathrm{r}}
$$

where $i$ distinguishes different fire regions and $\tau^{\mathrm{i}, \mathrm{r}}$ is an effective transmittance.

The camera measures $\mathrm{L}_{\mathrm{in}}^{\mathrm{i}, \mathrm{r}}$ and thus the radiance $\mathrm{L}_{\mathrm{em}}{ }^{\mathrm{i}, \mathrm{r}}$ emitted by a pixel of fire region $i$ in the spectral band $r$ can be obtained. This is only a fraction $\mathrm{F}$ of the radiation emitted in the full spectrum. Classification is necessary also in order to obtain this spectral fraction, since it depends on the spectral profile of the emitter:

$$
\mathrm{L}_{\mathrm{em}}^{\mathrm{i}, \mathrm{r}}=\mathrm{F}^{\mathrm{i}, \mathrm{r}} \cdot \mathrm{L}_{\mathrm{em}}^{\mathrm{i}, \text { total }}
$$

Finally, the total emitted radiation is given by:

$$
\mathrm{L}_{\mathrm{em}}^{\mathrm{i}, \text { total }}=\mathrm{L}_{\mathrm{in}}^{\mathrm{i}, \mathrm{r}} \cdot\left[\mathrm{F}^{\mathrm{i}, \mathrm{r}}\right]^{-1} \cdot\left[\tau^{\mathrm{i}, \mathrm{r}}\right]^{-1}
$$

\section{Classification of fire regions}

To classify a scene is to assign each pixel of the image to a specific "class", i.e., a region with physical properties relatively homogeneous. In fire studies, as previously explained, this is a necessary starting point for a region-specific processing that accounts for the heterogeneity of the scene.

The classification method used in this work is based on the differences of IR emission at two spectral bands $r_{1}$ and $r_{2}$. To classify a fire scene, the first step is to construct an " $r_{1}-r_{2}$ scatterplot". In this plot, each point stands for a pixel of the image, its ordinate and abscissa being, respectively, the incoming radiances in the $r_{1}$ and $r_{2}$ bands. For a complex scene, each region with more-or-less homogeneous radiative properties will generate a more-or-less compact cluster of points in the scatterplot. These are the "classes" into which we want to classify the scene.

As an example, Fig. 3 shows simultaneous images of a laboratory fire on a pine needles fuel bed (progressing from the bottom to the top of the image) at a distance of $7 \mathrm{~m}$. Images have been acquired with a Medium IR (MIR) camera that has a rotating four filter wheel. Filter F1 corresponds to the full band (1.5 to $5 \mu \mathrm{m}$ ), and the others are centered, respectively, at: F2 $=4,8 \mu \mathrm{m}, \mathrm{F} 3=4,2 \mu \mathrm{m}$, and $\mathrm{F} 4=3,6 \mu \mathrm{m}$, all of them with a full width at half maximum of $0,4 \mu \mathrm{m}$. The $\mathrm{F} 3$ band is much brighter in the flame region due to the strong $\mathrm{CO}_{2}$ emission.

The F3-F4 scatterplot is shown in Fig. 4 (axes have dimensions of radiances, in arbitrary units). Flame pixels form a cluster at the left-hand side, over the diagonal that corresponds to blackbody-like regions (embers and ashes). Several regions have been selected as typical of different classes and marked with different grey levels. The rest of the pixels (black dots in the scatterplot) are assigned to a class by a maximum likelihood algorithm [3]. As a result, the whole image becomes classified (Fig. 4, right).

Although there are a few classification errors (some pixels at the boundary of the flame are marked as ashes and other at its centre are labeled as fire front), on 
the whole the classification procedure just described is highly effective. It is also quite robust: calibration inaccuracies may alter the values of the pixels, but do not change appreciably the shape of the scatterplot and therefore do not alter significantly the classification results.
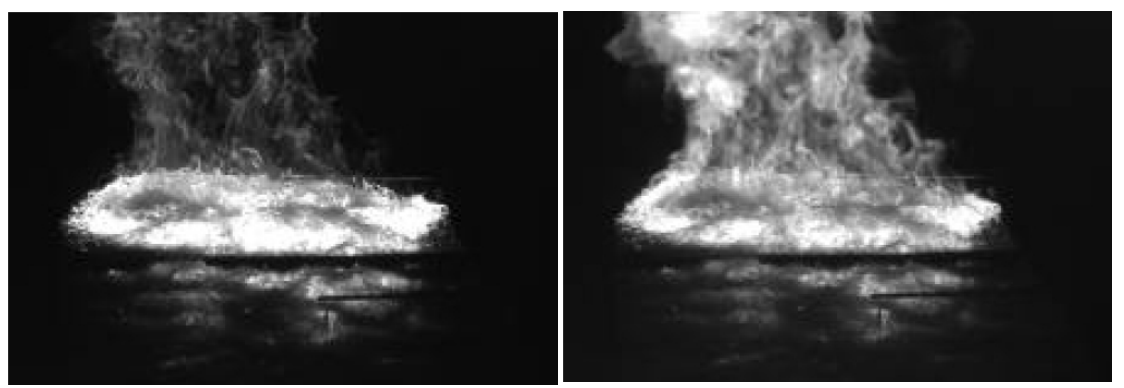

Figure 3: $\quad$ Images of a fire in the F4 band (left) and in the F3 band (right).

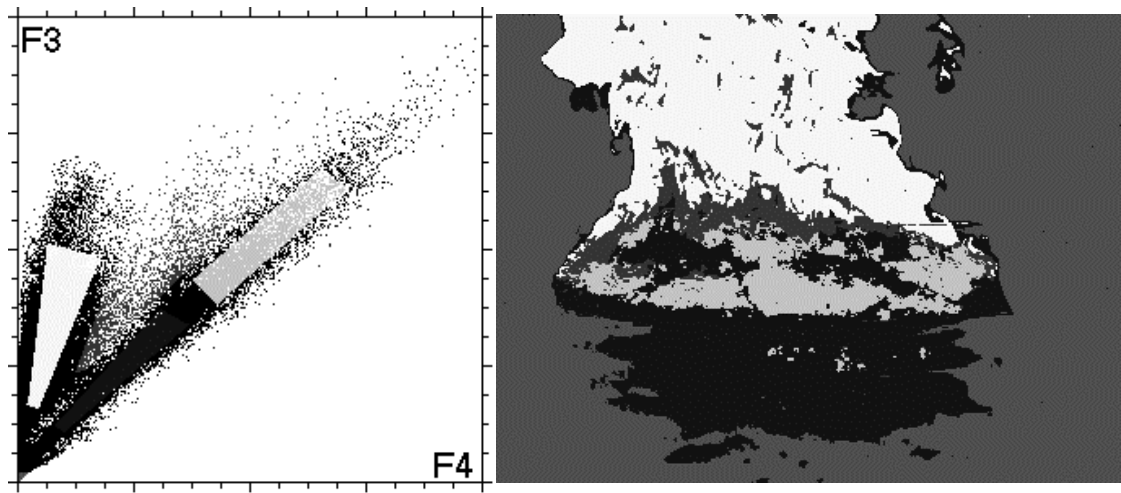

Figure 4: (Left) F3-F4 scatterplot for fig. 4. Each point represents a pixel. Several regions are marked with different grey levels. (Right) Image classified according to the regions marked on the scatterplot. The regions, in order of increasing brightness, are: cold embers, fire front, background, hot embers, flame.

Classification becomes more difficult as distance increases, because of atmospheric attenuation: atmospheric $\mathrm{CO}_{2}$ absorbs to a large degree the $\mathrm{CO}_{2}$ emission of the fire, thus blurring the distinction between flame and blackbodylike regions. In measurements from a distance of $550 \mathrm{~m}$, the F3-F4 scatterplot lost all the structure due to the important atmospheric absorption on the F3 band.

The best way to cope with this degradation is by using all the available bands (in our case, three, because F1 filter was saturated) with a technique called Principal Component Analysis (PCA). This is a standard method of image processing [3] that produces uncorrelated bands by making linear combinations of the often highly correlated original multispectral bands. There are as many PC 
bands as original bands, but most of the information is contained in the first two PC bands. In our case, PC1 is basically the overall brightness of the scene; whereas PC2 shows enhanced the flame regions and it provides a very useful mean to visualize fire progress (Fig. 5, left).

Figure 5 (right) shows values of PC2 and PC1 for typical regions of flame, fire front, embers and background obtained in several experimental fires. The ability to separate regions degrades with distance, but a good separation has been demonstrated up to at least $550 \mathrm{~m}$.
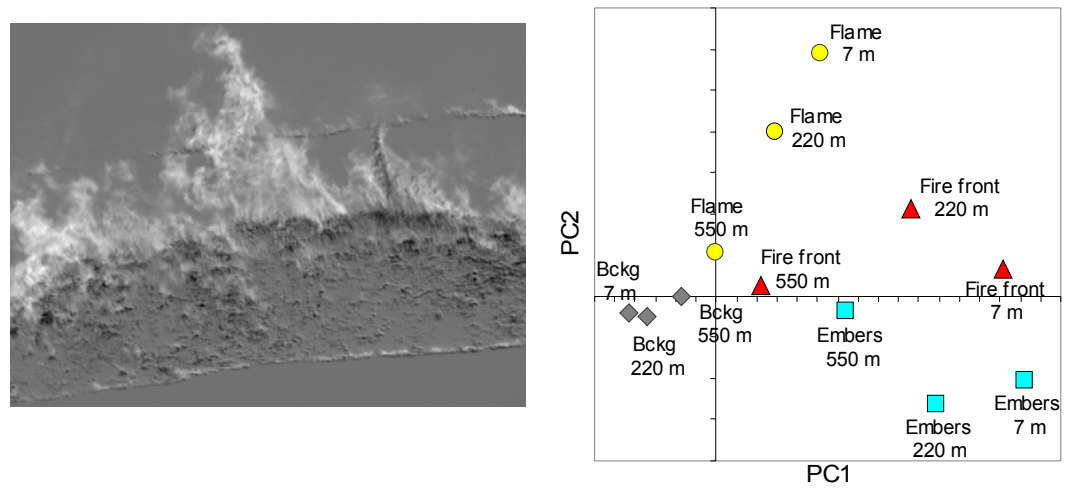

Figure 5: (Left) PC2 image for the scene of Fig. 1. (Right) Values of the first two principal components for several regions.

\section{Rate of spread and temperature measurements}

There are two methods to locate the fire front position with IR images. The simplest one is to use a band in which the flame signal is low (as the TIR band, or F4 band in Fig. 3) and identify the front by the temperature edge at the interface between embers and flame or background. The second method is to classify the whole sequence of a burn, obtaining a series of images in which the position of the flame/embers interface can be easily followed.

Both methods require a previous geo-referencing of the images; i.e., a "geometric calibration" in order to translate pixel coordinates into ground coordinates. This can be done by standard algorithms that use "ground control points" at places with known ground coordinates.

Figure 6 demonstrates that both IR methods agree with visual inspection for a laboratory fire measured at $7 \mathrm{~m}$ propagating linearly. The method based on brightness TIR temperature works well in this case, but there is no single value of temperature that works well for all forest fires. Classification is the most powerful methods and it can, in principle, be applied automatically by a software routine, although computation load is important.

The value of emitted radiation given by eqn (3) can be translated, according to Planck's law, into a temperature value called brightness temperature. However, this value will only agree with the true temperature for a blackbody. In 
a fire, the "solid" classes (embers and ashes) are blackbodies to a good approximation, but for a flame brightness MIR and TIR temperatures may differ markedly: the strong $\mathrm{CO}_{2}$ peak in the MIR region will make $\mathrm{T}_{\mathrm{MIR}}$ higher than $\mathrm{T}_{\mathrm{TIR}}$, although both temperatures will be lower than the real temperature of the flame (because flame emissivity is lower than one). Measurement of true flame temperatures is a complex problem, unsolved up to now by the IR image-based methods applicable in the field, but addressable in laboratory conditions [4].

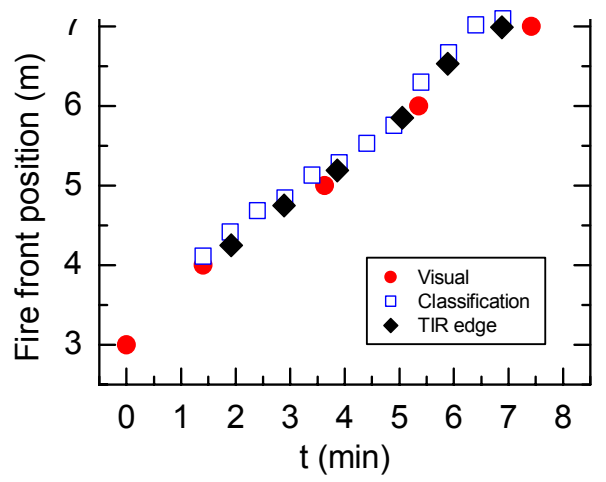

Figure 6: Fire front position versus time, as obtained by three different methods: visual inspection during the burn, classification, and time evolution of the TIR profile.

\section{Radiated power measurements}

Once classification has been performed, each pixel has been assigned to a class $i$ and therefore, using the corresponding factors $\mathrm{F}^{\mathrm{i}, \mathrm{r}}$ and $\tau^{\mathrm{i}, \mathrm{r}}$, the total radiance emitted by the pixel can be estimated by equation (3).

Factors $F^{i, r}$ and $\tau^{i, r}$ used in this work have been determined from the atmospheric transmittance profiles provided by MODTRAN software [5], the transmittance profiles of the camera filters, and the emission profiles measured with FTIR radiometry for each class in an experimental fire (see Fig. 2 for flame and nonflaming embers). For a distance of $550 \mathrm{~m}$, effective $\tau$ values vary from $13.1 \%$ (flame, F3) to $91.3 \%$ (embers, F4); F values range from 3.6\% (flame, F4) to $18.7 \%$ (flame, F3) [6].

To obtain the instantaneous radiative power of the fire requires the following steps:

1. Calculation of emitted MIR exitance $\left(\mathrm{W} / \mathrm{m}^{2}\right)$ for each pixel of each fire image. This is done by assuming lambertian radiation: values of radiance are simply multiplied by $\pi$.

2. Calculation of the area of each pixel, for each class. For the "solid" classes, this requires a previous geo-referencing of the images. The "flame" classes are considered to be in a vertical plane, and for them the ground slope is not considered in the calculation of pixel areas. On the 
other hand, its area is multiplied by an additional factor to take into account that only one side of the flame is seen (as a first approximation, a value of 2 has been used).

3. A pixel-per-pixel multiplication (exitance times area) of the previous images gives the power emitted by each pixel.

4. A spatial integration gives the power emitted by each class.
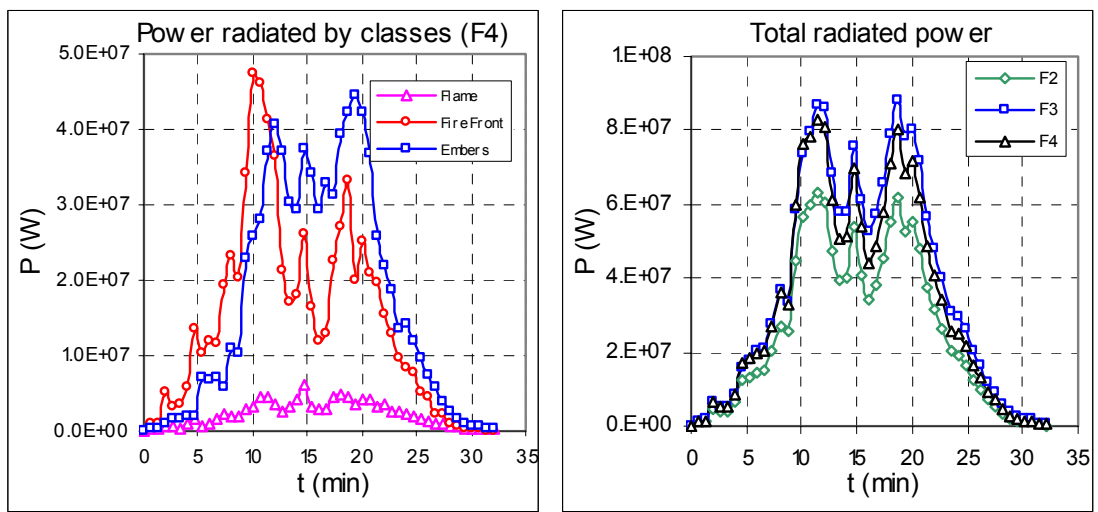

Figure 7: (Left) Power radiated for each class as a function of time, obtained from F4 images. (Right) Total power radiated by the fire.

Results for the fire at $550 \mathrm{~m}$ can be seen in Fig. 7. The left side shows the power radiated by the different classes, as obtained from F4 images. Total radiated energy, estimated from the three filters, is shown on the right side.

Table 1: $\quad$ Total radiated energy of the classes measured with the different filters and fraction radiated of total energy released

\begin{tabular}{|l|r|r|r|}
\hline & \multicolumn{1}{|l|}{ F2 } & \multicolumn{1}{l|}{ F3 } & F4 \\
\hline Embers & \multicolumn{1}{|l}{$26700 \mathrm{MJ}$} & $38900 \mathrm{MJ}$ & $32900 \mathrm{MJ}$ \\
\hline Fire front & $20000 \mathrm{MJ}$ & $28200 \mathrm{MJ}$ & $28100 \mathrm{MJ}$ \\
\hline Flame & $3300 \mathrm{MJ}$ & $4700 \mathrm{MJ}$ & $4300 \mathrm{MJ}$ \\
\hline Total & $50000 \mathrm{MJ}$ & $71800 \mathrm{MJ}$ & $65300 \mathrm{MJ}$ \\
\hline Fraction radiated & $11.3 \%$ & $16.2 \%$ & $14.7 \%$ \\
\hline
\end{tabular}

The total radiated energy is obtained by integration of the power curves. This energy is to be compared to the $444000 \mathrm{MJ}$ energy release estimated by fuel consumption (heat of combustion of fuel was $19135 \mathrm{~J} / \mathrm{g}$ ). Values are shown on Table 1.

If the corrections embodied in the $\tau$ and $\mathrm{F}$ parameters are correct, power estimations from the different filters should be equal. The discrepancies can be used as an estimation of the measurement error. Figure 7 and table 1 show that results from F3 and F4 are quite similar, but F2 gives smaller energy values. This may be a result of incorrect estimation of atmospheric transmittance. Since F4 
band is the less affected by the atmosphere, its value is the most reliable, and we can give $14 \pm 3 \%$ as the final value for fraction of released energy that is radiated (radiative fraction). This is in good agreement previous studies performed on small laboratory fires: a $17 \pm 3 \%$ value obtained by Aranda et al. [2] and a $14 \pm 3 \%$ value obtained by Wooster et al. [7].

It must be advised that, due to the extremely large dynamic range of the MIR images of forest fires, background is out of the measurement range and gives radiance values too large. An improved separation between cold embers and background classes may have an appreciable effect on fire power values, and it would be advisable to use an additional band in the thermal IR to refine classification of low temperature classes.

\section{Conclusions}

Measurements of rate of spread, embers temperature and radiated power from forest fires in the field have been demonstrated with multispectral ( 3 band) MIR images obtained at $550 \mathrm{~m}$. As a first step, a classification into different fire regions has been performed using Principal Component Analysis. Then, a region-specific processing provides emitted radiance at each point and time. A space integration gives instantaneous fire radiated power an a further time integration gives radiated energy. Comparison with released energy values estimated from fuel consumption data gives a value of $14 \pm 3 \%$ for the radiative fraction, in good agreement with previous results for small laboratory fires. Thus, fire power at each point can be obtained. A spatial integration gives then total power [Watts] and, subsequently, fire intensity [Watts/m]; and a time integration gives heat release per unit area [Joule $/ \mathrm{m}]$. All these parameters are obtained using only IR imaging remote measurements.

\section{Acknowledgements}

The authors wish to acknowledge C. Hernando, M. Guijarro and J. Madrigal for performing the short distance test at the CIFOR-INIA fire tunnel, in Madrid. Also, J. A. Vega and P. Cuiñas of CIF-Lourizan (Galicia) for the realization of the field tests. Finally, C. Van Bijleveld and F. Díaz for assistance in the measurements and image processing. This work has been partially funded by FIRESTAR project EVG1-CT-2001-00041.

\section{References}

[1] San-Miguel-Ayanz, J. \& Ravail, N., Active Fire Detection for Fire Emergency Management: Potential and Limitations for the Operational Use of Remote Sensing. Natural Hazards, 35, pp. 361-376, 2005.

[2] Aranda, J.M., Meléndez, J., de Castro, A.J., \& López, F., Bi-spectral Infrared Forest Fire Detection and Analysis Using Classification Techniques SPIE Proceedings, 5153, pp. 136-146, 2003. 
120 Modelling, Monitoring and Management of Forest Fires I

[3] J.A. Richards and X. Jia. Remote Sensing Digital Image Analysis, 3rd ed. Springer-Verlag, Berlin, 1999.

[4] Aranda, J.M., Briz, S., Meléndez, J, de Castro, A.J., and López, F., Flame analysis by IR thermography and hyperspectral imaging. Vth Quantitative Infrared Thermography QIRT 2000, Reims, 2000.

[5] Ontar Corporation, PcModWin3 Manual, Ontar Corporation, North Andover, Massachusetts, 1996.

[6] Meléndez, J., Aranda, J.M., de Castro, A.J., and López, F., Measurement of forest fire parameters with multi-spectral imaging in the medium infrared. QIRT Journal, 3, pp. 183-201, 2006.

[7] Wooster, M. J., Roberts, G., Perry, G.L.W., \& Kaufman, Y.J., Retrieval of biomass combustion rates and totals from fire radiative power observations: FRP derivation and calibration relationships between biomass consumption and fire radiative energy release. J. Geophys. Res.-Atmospheres 110 (D24): Art. No. D24311, 2005. 